\title{
Dietary fiber intake and glycemic control: coronary artery calcification in type 1 diabetes (CACTI) study
}

\author{
Arpita Basu ${ }^{1,2^{*}}$, Amy C. Alman ${ }^{1}$ and Janet K. Snell-Bergeon ${ }^{3}$
}

\begin{abstract}
Background: Dietary fiber has been recommended for glucose control, and typically low intakes are observed in the general population. The role of fiber in glycemic control in reported literature is inconsistent and few reports are available in populations with type 1 diabetes (T1D).

Methods: Using data from the Coronary Artery Calcification in Type 1 Diabetes (CACTI) study $[n=1257$; T1D: $n=$ 568; non-diabetic controls: $n=689]$ collected between March 2000 and April 2002, we examined cross-sectional (baseline) and longitudinal (six-year follow-up in 2006-2008) associations of dietary fiber and HbA1c. Participants completed a validated food frequency questionnaire, and a physical examination and fasting biochemical analyses ( $12 \mathrm{~h}$ fast) at baseline visit and at the year 6 visit. We used a linear regression model stratified by diabetes status, and adjusted for age, sex and total calories, and diabetes duration in the T1D group. We also examined correlations of dietary fiber with HbA1c.
\end{abstract}

Results: Baseline dietary fiber intake and serum HbA1c in the T1D group were $16 \mathrm{~g}$ [median (IQ): 11-22 g) and 7.9 $\pm 1.3 \%$ mean (SD), respectively, and in the non-diabetic controls were $15 \mathrm{~g}$ [median (IQ): 11-21 g) and 5.4 $\pm 0.4 \%$, respectively. Pearson partial correlation coefficients revealed a significant but weak inverse association of total dietary fiber with $\mathrm{HbA1c}$ when adjusted for age, sex, diabetes status and total calories $(r=-0.07, p=0.01)$. In the adjusted linear regression model at baseline, total dietary fiber revealed a significant inverse association with $\mathrm{HbA1c}$ in the T1D group $[\beta \pm \mathrm{SE}=-0.32 \pm 0.15, p=0.034]$, as well as in the non-diabetic controls $[-0.10 \pm 0.04, p=0.009]$. However, these results were attenuated after adjustment for dietary carbohydrates, fats and proteins, or for cholesterol and triglycerides. No such significance was observed at the year 6 follow-up, and with the HbA1c changes over 6 years.

Conclusion: Thus, at observed levels of intake, total dietary fiber reveals modest inverse associations with poor glycemic control. Future studies must further investigate the role of overall dietary quality adjusting for fiber-rich foods in T1D management.

Keywords: Dietary fiber, Hemoglobin A1c, Type 1 diabetes, Glycemia

\footnotetext{
* Correspondence: Arpita.basu@unlv.edu

${ }^{1}$ Epidemiology and Biostatistics, University of South Florida, Tampa, USA

${ }^{2}$ Department of Kinesiology and Nutrition Sciences, University of Nevada Las

Vegas, Las Vegas, USA

Full list of author information is available at the end of the article
}

(c) The Author(s). 2019 Open Access This article is distributed under the terms of the Creative Commons Attribution 4.0 International License (http://creativecommons.org/licenses/by/4.0/), which permits unrestricted use, distribution, and reproduction in any medium, provided you give appropriate credit to the original author(s) and the source, provide a link to the Creative Commons license, and indicate if changes were made. The Creative Commons Public Domain Dedication waiver (http://creativecommons.org/publicdomain/zero/1.0/) applies to the data made available in this article, unless otherwise stated. 


\section{Background}

The incidence of type 1 diabetes (T1D), an autoimmune disorder, as well as cardiovascular disease (CVD), the major vascular complication of diabetes have been increasing worldwide [1, 2]. Based on the statistics reported by the American Heart Association, only 1.5\% of US adults meet the guidelines for healthy diet pattern [2]. While the recommendation of healthy dietary pattern may have poor compliance in populations, the role of modifying individual nutrients and bioactive compounds has gained much attention in the management of chronic diseases such as T1D. Among the dietary components, fiber has been shown to play an important role in glycemic control in diabetes [3]. In a meta-analysis of randomized clinical trials reported by Silva et al. (2013), higher fiber diets (up to $42.5 \mathrm{~g} /$ day) or supplements containing soluble fiber ( $15 \mathrm{~g} /$ day) were found to significantly decrease HbA1c and fasting plasma glucose in adults with type 2 diabetes [4]. In another systematic review, foods rich in soluble fiber, such as beta-glucans, were shown to improve glycemia in diabetes patients [5]. These effects of fiber have been explained by biological mechanisms in delaying gastric emptying and decreasing glucose absorption that subsequently lead to decreases in postprandial rise of blood glucose $[6,7]$. In addition, dietary fiber, especially increased soluble fiber intake, has also been associated with anti-inflammatory properties and in modulating the immune system with potential implications in the prevention of T1D in children [8].

Epidemiological evidence on the associations of dietary fiber and glycemic control in adults with type 1 diabetes is limited and conflicting. In a longitudinal study of youths with type 1 diabetes $(n=136)$, following behavioral nutrition intervention, fiber intake was associated with improved glycemic control [9]. Data from the European Diabetes Centers (EURODIAB) Prospective Complications Study have reported lower fiber intake in adults with type 1 diabetes $(n=1102)$ [10], as well as an inverse association of fiber with HbA1c in these adults $(n=1659)$ [11]. In another study, fiber intake was not associated with HbA1c control in youths $(n=908)$ in a longitudinal dietary study [12]. While several factors may be involved in the differences in these study findings, participant characteristics, such as levels of dietary fiber intake and cardio-metabolic profiles, and duration of diabetes may play an important role, and thus a need to further investigate these associations in well-defined cohorts of T1D.

We have previously reported low prevalence of ideal cardiovascular health (1.1\%), especially based on the American Heart Association definition of health matrices, in adults with type 1 diabetes in the coronary artery calcification in type 1 diabetes (CACTI) study [13]. We now aim to identify the associations of dietary fiber with glycemic control in the same cohort at cross-sectional and longitudinal time points.

\section{Methods}

\section{Study participants}

The data presented in this report were collected as part of the baseline examination of the CACTI study. The study enrolled 1416 individuals between 19 and 56 years of age, with no known history of CHD: 652 subjects with type 1 diabetes and 764 nondiabetic control subjects. Participants with type 1 diabetes had long-standing disease (mean duration 23 years, range 4-52 years), were insulin dependent within a year of diagnosis, and were diagnosed prior to age 30 or had positive antibodies or a clinical course consistent with type 1 diabetes. Non-diabetic control subjects had fasting blood glucose $<110 \mathrm{mg} / \mathrm{dL}$ and were generally spouses, friends and neighbors of cases. The inclusion and exclusion criteria have been described previously [14]. All study participants provided informed consent and the study protocol was approved by the Colorado Multiple Institutional Review Board.

\section{Dietary intake}

Study participants who completed the baseline screening visit were asked to fill out a validated self-administered semi quantitative food-frequency questionnaire of 126 food items (Harvard FFQ, 1988) [15]. The procedures have been previously published in detail [14]. The nutrient values and dietary fiber content of foods for the Harvard FFQ were estimated primarily on the basis of the USDA food composition database, and the correlation coefficient for dietary fiber was 0.68 based on the validation studies $[15,16]$. The total dietary fiber content was mainly derived from the following food groups included in the FFQ: breakfast cereals, breads, other cereal foods, potatoes, legumes, lentils, vegetables, fruits, and nuts and seeds. One thousand three hundred six study participants completed the FFQ. Calorie intake was calculated per the guidelines suggested by Willett [17] for excluding individuals with implausible reported energy intake, 40 participants were excluded due to reported caloric intake that was very low $(<500 \mathrm{cal}$ per day if female or $<$ $800 \mathrm{cal}$ per day if male), or very high (3500 or higher if female, 4000 or higher if male), leaving 571 participants with type 1 diabetes and 696 controls in this analysis [14]. Among these, for the current analysis, complete biochemical and dietary information was available in 568 participants with type 1 diabetes and 689 controls and were included in the final cross-sectional analysis.

\section{Cardiovascular risk factors}

Participants completed a baseline examination between March 2000 and April 2002. Anthropometric measurements were obtained and included height, weight and waist circumference. Body mass index was calculated in $\mathrm{kg} / \mathrm{m}^{2}$. Resting systolic blood pressure (SBP) and fifth-phase diastolic blood pressure (DBP) were measured three times 
while the patients were seated, following a $5 \mathrm{~min}$ rest, and the second and third measurements were averaged (Omron HEM-705CP). In addition, participants completed standardized questionnaires that enquired about medical history, current medication, insulin doses, physical activity, alcohol and tobacco use and family medical history. Following a $12 \mathrm{~h}$ fast, participants came to the clinic in the morning for blood collection and analyses of biochemical variables. Lipids (total cholesterol, HDL-cholesterol, LDLcholesterol and triglycerides), fasting glucose and HbA1c were measured. After an overnight fast, blood was collected, centrifuged and separated. Plasma was stored at $4{ }^{\circ} \mathrm{C}$ until assayed. Total plasma cholesterol and triglyceride levels were measured using standard enzymatic methods; HDL cholesterol was separated using dextran sulphate, and LDL cholesterol was calculated using the Friedewald formula. High-performance liquid chromatography was used to measure $\mathrm{HbA}_{1 \mathrm{c}}$ (HPLC, BioRad variant).

\section{Statistical analysis}

Variables were examined for normality (normal plots), and non-normally distributed variables (dietary fiber, plasma triglycerides) were log transformed. Differences in risk factors between men and women with type 1 diabetes and without diabetes were examined using a Student's t test. A $\chi 2$ test for goodness of fit was used to determine if categorical risk factors differed between patients with type 1 diabetes and non-diabetic participants. Wilcoxon rank sum test was used to compare differences of continuous variables with skewed distributions. Correlations of dietary fiber with HbA1c, and dietary macronutrients (carbohydrates, total fats and proteins as percentage of daily caloric intake) and cardiovascular risk factors (systolic and diastolic blood pressure, BMI, waist circumference, plasma total-, HDL- and LDL-cholesterol and triglycerides, and glucose) were examined using Pearson correlation coefficients after adjusting for age, sex, diabetes status and total calories in the entire cohort, as well as by T1D status. Linear regression analysis was used to examine associations of dietary fiber intake at baseline with contemporaneous $\mathrm{HbA1c}$, as well as with HbA1c at year 6 follow-up and the change between year 6 and baseline. Models were adjusted for relevant covariates as follows: model 1 (age, sex, and total calories, and diabetes duration for T1D), model 2 (model $1+$ dietary carbohydrates, fats and proteins) and model 3 (model $1+$ plasma lipids). In addition, longitudinal analyses were adjusted for baseline $\mathrm{HbA1c}$ and the duration of follow-up. Logistic regression analysis was used to examine associations of quintiles of total dietary fiber with the probability of poor control (>7\%) vs. optimal control $(<7 \%)$ of HbA1c at baseline and the 6-year follow-up.

\section{Results}

A total of 1257 participants were included in the cross-sectional analysis, and a total of 990 participants who had $\mathrm{HbA1c}$ values at both baseline and Year 3 were included in the longitudinal analysis in this report. Table 1 shows the baseline characteristics of the study participants stratified by sex and diabetes status. Women were younger than men in both groups, significantly so in the non-diabetic control group. Among the anthropometric and biochemical measures in the T1D group, BMI, waist circumference, systolic and diastolic blood pressure were significantly lower in women, while HDL-cholesterol was higher when compared to men. We observed similar differences in the control group, in addition to significantly lower fasting glucose, total cholesterol and triglycerides in women than in men. Among the dietary nutrient intakes, total energy intake was significantly lower, while carbohydrate and protein intake were modestly higher in women than men in both groups. No significant differences in total fiber intake were observed between men and women in the T1D or control group (Table 1).

Table 2 shows adjusted correlation coefficients of dietary fiber intake with anthropometrics, biochemical and dietary variables in the entire cohort, as well as by diabetes status. Dietary fiber exhibited a significant and inverse correlation with $\mathrm{HbA1c}$, BMI, waist circumference, systolic and diastolic blood pressure, serum cholesterol and triglyceride. Among the dietary nutrients, fiber intake revealed a significant positive association with total carbohydrates, and an inverse association with total fat intake in the entire cohort (all $p<0.05$ ). These significant correlations persisted in non-diabetic controls, but were somewhat attenuated in T1D cases for BMI, waist circumference, plasma HDL-C, triglycerides and glucose. Overall, total dietary fiber intake remained inversely correlated with HbA1c in T1D cases as well as non-diabetic controls (Table 2).

Table 3 shows the associations of baseline dietary total fiber intake as a continuous variable with $\mathrm{HbA1c}$ at year 6 and the change (year 6 - baseline) stratified by diabetes status. Cross-sectional analysis at baseline revealed a significant inverse association of dietary fiber intake with HbA1c in the model adjusted for age, sex, total calories and diabetes duration in the T1D group, as well as in non-diabetic controls (Model 1). The significance did not persist in models further adjusted for dietary nutrients (Model 2) and conventional lipids (Model 3). Longitudinal analyses revealed no significant association in any of the models examined. The six-year change in HbA1c was observed to be 0.05 ($0.69-0.63) \%$, median (IQR) in the T1D group, and 0.01 $(-0.2-0.30) \%$, in the non-diabetic controls. Log-transformed values of fiber were used for analyses presented in Tables 2 and 3. 
Table 1 Baseline characteristics of the CACTI cohort

\begin{tabular}{|c|c|c|c|c|c|c|}
\hline & Type 1 diabetes & & & Non-diabetic control & & \\
\hline & Men $(n=251)$ & Women $(n=320)$ & $p$-value & Men $(n=347)$ & Women $(n=349)$ & $p$-value \\
\hline Age (years) & $38.0 \pm 9.0$ & $36.0 \pm 9.0$ & 0.07 & $40.0 \pm 9.0$ & $38.0 \pm 9.0$ & 0.01 \\
\hline HbA1c (\%) & $7.9 \pm 1.2$ & $7.9 \pm 1.3$ & 0.75 & $5.6 \pm 0.4$ & $5.4 \pm 0.4$ & $<0.0001$ \\
\hline Met < 7\% HbA1c goal (\%) & 18.7 & 22.0 & 0.67 & N/A & N/A & N/A \\
\hline Duration of diabetes (years) & $24.0 \pm 9.0$ & $23.0 \pm 9.0$ & 0.18 & N/A & N/A & N/A \\
\hline Duration of follow-up (years) & $6.2 \pm 0.6$ & $6.2 \pm 0.5$ & 0.23 & $6.1 \pm 0.5$ & $6.2 \pm 0.6$ & 0.25 \\
\hline BMI $\left(\mathrm{kg} / \mathrm{m}^{2}\right)$ & $26.6 \pm 3.8$ & $25.8 \pm 4.7$ & 0.04 & $27.2 \pm 4.1$ & $25.1 \pm 5.6$ & $<0.0001$ \\
\hline Waist circumference (cm) & $90.8 \pm 11.0$ & $81.0 \pm 12.0$ & $<0.0001$ & $93.0 \pm 12.0$ & $79.0 \pm 13.0$ & $<0.0001$ \\
\hline Systolic blood pressure (mm Hg) & $122.0 \pm 13.0$ & $114.0 \pm 14.0$ & $<0.0001$ & $118.0 \pm 11.0$ & $111.0 \pm 13.0$ & $<0.0001$ \\
\hline Diastolic blood pressure (mm Hg) & $80.0 \pm 9.0$ & $75.0 \pm 8.0$ & $<0.0001$ & $82.0 \pm 8.0$ & $76.0 \pm 8.0$ & $<0.0001$ \\
\hline Plasma glucose (mg/dL) & $199.0 \pm 102.0$ & $187.0 \pm 93.0$ & 0.14 & $93.0 \pm 10.0$ & $87.0 \pm 9.0$ & $<0.0001$ \\
\hline Plasma cholesterol (mg/dL) & $175.0 \pm 35.0$ & $176.0 \pm 33.0$ & 0.81 & $198.0 \pm 43.0$ & $185.0 \pm 34.0$ & $<0.0001$ \\
\hline Plasma HDL-cholesterol (mg/dL) & $50.0 \pm 13.0$ & $60.0 \pm 17.0$ & $<0.0001$ & $43.0 \pm 11.0$ & $58.0 \pm 14.0$ & $<0.0001$ \\
\hline Plasma triglycerides (mg/dL) & $83.0(62.0-114.0)$ & $77.0(62.0-103.0)$ & 0.08 & $123.0(89.0-181.0)$ & $89.0(66.0-124.0)$ & $<0.0001$ \\
\hline Energy intake (kcal/day) & $1954.0 \pm 625.0$ & $1633.0 \pm 561.0$ & $<0.0001$ & $1992.0 \pm 655.0$ & $1655.0 \pm 528.0$ & $<0.0001$ \\
\hline Carbohydrate intake (\% kcal/day) & $44.0(38.0-51.0)$ & $46.0(40.0-52.0)$ & 0.04 & $47.0(42.0-52.0)$ & $48.0(42.0-54.0)$ & 0.04 \\
\hline Fat intake (\% kcal/day) & $36.0(31.0-41.0)$ & $35.0(30.0-39.0)$ & 0.08 & $34.0(29.0-37.0)$ & $33.0(28.0-36.0)$ & 0.06 \\
\hline Protein intake (\% kcal/day) & $18.0(16.0-21.0)$ & $19.0(17.0-21.0)$ & 0.004 & $18.0(15.0-20.0)$ & $19.0(16.0-21.0)$ & 0.0004 \\
\hline Total dietary fiber (g) & $16.0(12.0-22.0)$ & $15.0(11.0-21.0)$ & 0.06 & $15.0(11.0-21.0)$ & $16.0(11.0-21.0)$ & 0.88 \\
\hline Physical activity (kJ/week) & 7517 (3165-14,118) & $5020(1938-10,862)$ & 0.32 & $6986(3048-12,861)$ & $6232(2859-11,255)$ & 0.21 \\
\hline
\end{tabular}

Data are presented as means \pm SD and median (IQ range)

$P<0.05$ in bold; Comparison between men and women: $t$ test for difference in means, $\mathrm{X} 2$ test for difference in proportions, and Wilcoxon rank sum test for difference of continuous variables with skewed distributions

Table 2 Pearson partial correlation coefficients of total dietary fiber with clinical parameters and dietary variables in the CACTI cohort

\begin{tabular}{|c|c|c|c|c|c|c|}
\hline \multirow[t]{2}{*}{ Variable } & \multicolumn{2}{|c|}{$\begin{array}{l}\text { Total dietary fiber (all subjects) } \\
(n=1257)\end{array}$} & \multicolumn{2}{|c|}{$\begin{array}{l}\text { Total dietary Fiber T1D cases } \\
(n=568)\end{array}$} & \multicolumn{2}{|c|}{$\begin{array}{l}\text { Total dietary fiber non-diabetic controls } \\
(n=689)\end{array}$} \\
\hline & $\bar{R}$ & $p$-value & $\mathrm{R}$ & $p$-value & $\mathrm{R}$ & $p$-value \\
\hline $\mathrm{HbA1c}$ & -0.07 & 0.01 & -0.08 & 0.03 & -0.10 & 0.009 \\
\hline Systolic blood pressure & -0.11 & 0.0001 & -0.08 & 0.05 & -0.12 & 0.002 \\
\hline Diastolic blood pressure & -0.13 & $<0.0001$ & -0.09 & 0.03 & -0.13 & 0.001 \\
\hline BMI & -0.14 & $<0.0001$ & -0.02 & 0.56 & -0.16 & $<0.0001$ \\
\hline Waist circumference & -0.12 & $<0.0001$ & -0.01 & 0.73 & -0.14 & 0.0003 \\
\hline Plasma cholesterol & -0.09 & 0.0007 & -0.12 & 0.005 & -0.06 & 0.11 \\
\hline Plasma HDL- cholesterol & 0.04 & 0.16 & -0.02 & 0.56 & 0.11 & 0.004 \\
\hline Plasma triglyceride & -0.07 & 0.009 & -0.008 & 0.85 & -0.10 & 0.007 \\
\hline Plasma glucose & -0.03 & 0.33 & -0.02 & 0.59 & -0.08 & 0.022 \\
\hline Carbohydrate (\% daily intake) & 0.43 & $<0.0001$ & 0.45 & $<0.0001$ & 0.43 & $<0.0001$ \\
\hline Protein intake (\% daily intake) & -0.05 & 0.089 & -0.08 & 0.05 & -0.04 & 0.26 \\
\hline Fat intake (\% daily intake) & -0.41 & $<0.0001$ & -0.40 & $<0.0001$ & -0.43 & $<0.0001$ \\
\hline
\end{tabular}

Based on log transformed values of dietary fiber

Adjusted for age, sex, total calories and diabetes status, and duration (T1D)

T1D type 1 diabetes

$P<0.05$ in bold 
Table 3 Linear associations of total dietary fiber with $\mathrm{HbA1c}$ at baseline, prospective (year 6) and change data in the CACTI cohort

\begin{tabular}{|c|c|c|c|c|c|c|c|c|c|c|c|c|}
\hline \multirow{3}{*}{$\begin{array}{l}\text { Dietary } \\
\text { fiber } \\
\text { (Baseline) }\end{array}$} & \multicolumn{4}{|c|}{ HbA1c (Baseline) } & \multicolumn{4}{|c|}{$\mathrm{HbA1c}$ (year 6) } & \multicolumn{4}{|c|}{ HbA1c change (year 6-Baseline) } \\
\hline & \multicolumn{2}{|c|}{$\begin{array}{l}\mathrm{T1D} \\
(n=568)\end{array}$} & \multicolumn{2}{|c|}{$\begin{array}{l}\text { Non-diabetic control } \\
(n=689)\end{array}$} & \multicolumn{2}{|c|}{$\mathrm{T1D}(n=452)$} & \multicolumn{2}{|c|}{$\begin{array}{l}\text { Non-diabetic control } \\
(n=538)\end{array}$} & \multicolumn{2}{|c|}{$\mathrm{T1D}(n=452)$} & \multicolumn{2}{|c|}{$\begin{array}{l}\text { Non-diabetic control } \\
(n=538)\end{array}$} \\
\hline & beta $\pm S E$ & $P$-value & beta \pm SE & $P$-value & beta \pm SE & $P$-value & beta $\pm S E$ & $P$-value & beta \pm SE & $P$-value & beta \pm SE & $P$-value \\
\hline Model $1^{\mathrm{a}}$ & $-0.32 \pm 0.15$ & 0.034 & $-0.10 \pm 0.04$ & 0.009 & $0.017 \pm 0.138$ & 0.90 & $0.033 \pm 0.057$ & 0.56 & $0.017 \pm 0.138$ & 0.90 & $0.033 \pm 0.057$ & 0.56 \\
\hline Model $2^{\mathrm{b}}$ & $-0.14 \pm 0.18$ & 0.43 & $-0.06 \pm 0.05$ & 0.27 & $0.112 \pm 0.158$ & 0.48 & $0.044 \pm 0.065$ & 0.50 & $0.112 \pm 0.158$ & 0.48 & $0.044 \pm 0.065$ & 0.50 \\
\hline Model $3^{c}$ & $-0.08 \pm 0.11$ & 0.46 & $-0.05 \pm 0.03$ & 0.19 & $0.119 \pm 0.158$ & 0.45 & $0.058 \pm 0.065$ & 0.37 & $0.119 \pm 0.158$ & 0.45 & $0.058 \pm 0.065$ & 0.37 \\
\hline
\end{tabular}

T1D type 1 diabetes

$P<0.05$ are in bold font

${ }^{a}$ Model adjusted for age, sex, diabetes duration and total calories for T1D; age, sex and total calories for non-diabetic control; year 6 and change model also adjusted for baseline HbA1c and duration of follow-up

${ }^{\mathrm{b}}$ Model $1+$ dietary carbohydrates, fats and proteins

${ }^{c}$ Model $1+$ plasma total cholesterol and triglycerides

Table 4 shows data from logistic regression analysis to examine the associations of quintiles of dietary fiber intake with the probability of poor vs. optimal glycemic control (HbA1c > 7\% vs. $<7 \%)$. At baseline, no significant associations were noted, but those in the highest category of fiber intake [29 (23-69 g), mean (range)] compared to the lowest (reference), trended towards an inverse association in adjusted analysis $(p=0.08$, Table 4$)$. No significant associations were noted at year 6 follow-up. No significant interaction effects of dietary fiber and sex were noted in our analyses $(p=0.22)$.

\section{Discussion}

To our knowledge few reports have been published on the association between dietary fiber intake and glycemic control in T1D. Thus, our study found a significant inverse association between total dietary fiber intake and HbA1c levels at the baseline visit of the CACTI study in those with diabetes, as well as in non-diabetic controls in a model adjusted for standard covariates including total calories. Our significant cross-sectional associations of fiber with glycemic control did not persist in models further adjusted for dietary macronutrients and blood lipids, largely due to their known independent associations with glycemic control $[18,19]$. We did not observe any significant predictive association of baseline fiber intake with HbA1c levels at year 6, and the six-year changes of HbA1c in adjusted models. These discrepancies in observations between cross-sectional and prospective associations may be explained by the smaller sample size in our prospective analysis and the habitual low baseline fiber intake that was not predictive of glycemic control 6 years later. This argument may further explain our observation of an inverse trend between fiber intake and poorly controlled HbA1c (>7\%) but only in the highest quintile of fiber intake in the T1D group. Poor glycemic control in T1D has been associated with excess mortality and cardiovascular disease (CVD) when compared to non-diabetic matched controls [20, 21], and few adults with T1D meet current glycemic control targets [20], thus necessitating additional preventive strategies such as dietary fiber intake in this high-risk population. Dietary fiber has been identified as a nutrient of public health concern and most of the US adults do not meet the recommendations of $38 \mathrm{~g} /$ day for adults [22]. We observed an intake of dietary fiber of less than half the recommendations in our cohort of individuals with and without T1D. These findings in adults may also be reflective of a continuum of poor dietary fiber intake observed in children with T1D [23], thus

Table 4 Logistic regression associations of quintiles of total dietary fiber with the probability of poor control (> 7\%) vs. optimal control (<7\%) HbA1c at baseline and at year 6 of CACTI study

\begin{tabular}{lllll}
\hline $\begin{array}{l}\text { Dietary fiber } \\
\text { quintiles (g) }\end{array}$ & $\begin{array}{l}\text { HbA1c goals (baseline) } \\
(>7 \% N=453 \text { vs. }<7 \% N=115)\end{array}$ & & & $\begin{array}{l}\text { HbA1c goals (year 6) } \\
(>7 \% N=361 \text { vs. }<7 \% N=91)\end{array}$ \\
\cline { 2 - 3 } & OR $(95 \% \mathrm{Cl})$ & $P$-value & & Reference \\
\hline Quintile 1 & Reference & 0.24 & $1.197(0.546,2.625)$ & $P$-value \\
Quintile 2 & $0.487(0.147,1.617)$ & 0.16 & $1.059(0.475,2.359)$ & 0.65 \\
Quintile 3 & $0.420(0.126,1.407)$ & 0.15 & $1.987(0.787,5.021)$ & 0.88 \\
Quintile 4 & $0.370(0.096,1.423)$ & 0.08 & $1.573(0.559,4.432)$ & 0.15 \\
Quintile 5 & $0.283(0.07,1.153)$ & &
\end{tabular}

Data presented as OR $(95 \% \mathrm{Cl})$

Dietary fiber quintiles: Quintile 1[N: 253; mean: $7.8 \mathrm{~g}$ (range: 2.8-10.3 g)]; Quintile 2 [N: 254; mean: $12.0 \mathrm{~g}$ (range: 10.3-13.7 g)]; Quintile 3 [N: 253 ; mean: $15.5 \mathrm{~g}$ (range: 13.7-17.4 g)]; Quintile 4 [N: 254; mean: $19.8 \mathrm{~g}$ (range: 17.4-22.7 g)]; Quintile 5 [N: 253; mean: $28.8 \mathrm{~g}$ (range: 22.7-68.5 g)]

Model adjusted for age, sex, diabetes duration and total calories; year 6 also adjusted for baseline HbA1c and duration of follow up 
identifying a strong need to improve dietary fiber intake in early life in T1D populations.

Very few studies have been reported on the association of dietary fiber with glycemic control, especially in T1D. Our study findings agree with a few previously reported studies showing no prospective associations of dietary fiber with HbA1c in youths with T1D $[9,12]$, while, we observe similar findings of inverse cross-sectional association between dietary fiber and HbA1c in adults with T1D in the EURODIAB study [24], as well as in youths with T1D [25]. Our longitudinal findings differ from another report from the EURODIAB study in which baseline fiber intake revealed significant protective association against elevated HbA1c levels in a 6.8 year follow-up period [11]. In comparing our study with this previously reported study, we observe some differences, especially higher $\mathrm{HbA1c}$ at baseline and follow-up years, $8.25 \pm 1.85 \%$ and $8.27 \pm 1.44 \%$ (mean \pm SD), respectively, in their study [11], vs. $7.9 \pm 1.2 \%$ and 7.8 $\pm 1.12 \%$, respectively, in our study. Also, our smaller sample size at the six-year follow-up $(n=990)$, when compared to this previous study $(n=1659)$ could explain the null findings in our longitudinal analysis. Further, there are numerous factors that impact changes in glycemic control over time, including adoption of new diabetes technology, such as the use of diabetes apps and remote glucose monitoring system [26] during the study period. Overall, the importance of increasing consumption of dietary fiber must be emphasized in the T1D population, based on the numerous health benefits of dietary fiber in delaying gastric transit time and improving postprandial glucose load, decreasing inflammation and cholesterol levels [27-29].

Our findings of the inverse association of dietary fiber with $\mathrm{HbA} 1 \mathrm{c}$ in the non-diabetic controls agree with previous reports in such populations, and have implications for reducing risks associated with obesity, the metabolic syndrome and type 2 diabetes in the general population [30-33]. In addition to our main outcome of HbA1c, we also observed significant inverse correlations of dietary fiber with BMI, waist circumference, systolic and diastolic blood pressure, as well as serum cholesterol and triglycerides. These findings conform to reported studies on the protective associations of dietary fiber against obesity, the metabolic syndrome and elevated blood lipids [31, 32]. We also observed a moderately strong and significant inverse association between dietary fiber and total fat intake in the entire cohort, and this provides some evidence of unhealthy dietary patterns in T1D populations [34]. We have previously reported higher intake of total and saturated fats in the CACTI cohort that were associated with poor glycemic control [14]. Together with our current findings on fiber intake, selected nutrients, such as dietary fats, and dietary bioactive compounds may play an important role in glycemic control in T1D.
Our analyses have some limitations that must be considered during the interpretation of results. In the first place, nutritional exposure data at baseline dietary intakes from the FFQ relied on a retrospective self-report and, therefore, may have been prone to recall bias. Secondly, we did not have information on the distribution of soluble vs. insoluble fiber intake, food groups contributing to total fiber intake, as well as food bioactive compounds, such as resistant starch shown to be associated with improved glycemic mangement $[9,35,36]$. Thirdly, our study considered HbA1c as a marker of long-term glycemic control, and we did not have data on daily glucose measures to capture day-to-day glycemic fluctuations, which have recently been suggested as a strong predictor of overall glycemic control [37]. Future prospective cohort studies should thus examine associations of dietary fiber intake from different food groups, as well as different types of fiber using multiple biomarkers of glycemic control.

\section{Conclusions}

In conclusion, our significant inverse association observed between dietary fiber intake and $\mathrm{HbAlc}$ in the model adjusted for age, sex and total calories, and diabetes duration for T1D at baseline visit (cross-sectional analysis) provides some evidence on the role of fiber intake in glycemic control, which is of importance in the management of T1D patients at a high risk of mortality from CVD. This association did not persist in models further adjusted for dietary macronutrients and plasma total cholesterol and triglycerides, and this may indicate that higher levels of fiber intake than the observed low habitual intakes are needed to counteract the positive associations of these variables with HbA1c. Overall, our adjusted correlation coefficients also revealed a significant inverse association of fiber intake with $\mathrm{HbAlc}$ in the entire cohort, as well as in T1D cases and non-diabetic controls. We used data from a well-characterized cohort of T1D patients as well as matched non-diabetic controls, thereby permitting generalizability of our data to these populations. Further research may explore whether overall dietary quality when adjusted for fiber intake is associated with glycemic control in participants with T1D or non-diabetic individuals with habitual low fiber intakes.

\footnotetext{
Acknowledgements

We would like to thank all participants for their contributions to this study.

Funding

Support for this study was provided by the National Institutes of Health National Heart, Lung and Blood Institute grants R01 HL61753, R01 HL079611 and R01 HL113029, American Diabetes Association grant 7-06-CVD-28, American Diabetes Association Grant 7-13-CD-10 (Snell-Bergeon) and Diabetes Endocrinology Research Center Clinical Investigation Core P30 DK57516. The study was performed at the Adult General Clinical Research Center at the University of Colorado Denver Anschutz Medical Center supported by the $\mathrm{NIH}$ M01 RR000051 and NIH/NCATS Colorado CTSA Grant Number UL1
} 
TR002535, the Barbara Davis Center for Childhood Diabetes in Denver and at Colorado Heart Imaging Center in Denver, CO, USA.

\section{Availability of data and materials \\ No applicable}

\section{Authors' contributions}

$A B, A C A$ and JKS-B contributed to the conception and design of the study, analysis, and interpretation of the data and drafted the manuscript. All authors critically revised, read, and approved the final manuscript and agreed to be fully accountable for ensuring the integrity and accuracy of the work.

\section{Ethics approval and consent to participate}

All study participants provided informed consent and the study protocol was approved by the Colorado Multiple Institutional Review Board. This observational study was performed in consistent with the approved guidelines.

\section{Consent for publication}

Not applicable.

\section{Competing interests}

The authors declare that they have no competing interests.

\section{Publisher's Note}

Springer Nature remains neutral with regard to jurisdictional claims in published maps and institutional affiliations.

\section{Author details}

'Epidemiology and Biostatistics, University of South Florida, Tampa, USA. ${ }^{2}$ Department of Kinesiology and Nutrition Sciences, University of Nevada Las Vegas, Las Vegas, USA. ${ }^{3}$ Barbara Davis Center for Childhood Diabetes, University of Colorado, Anschutz Medical Campus, Aurora, USA.

Received: 1 November 2018 Accepted: 26 March 2019

Published online: 03 April 2019

\section{References}

1. Forlenza GP, Rewers M. The epidemic of type 1 diabetes: what is it telling us? Curr Opin Endocrinol Diabetes Obes. 2011;18(4):248-51. https://doi.org/ 10.1097/MED.0b013e32834872ce [published Online First: 2011/08/17].

2. Mozaffarian D, Benjamin EJ, Go AS, et al. Executive summary: heart disease and stroke statistics--2016 update: a report from the American Heart Association. Circulation. 2016;133(4):447-54. https://doi.org/10.1161/cir. 0000000000000366 [published Online First: 2016/01/27].

3. Weickert MO, Pfeiffer AFH. Impact of dietary fiber consumption on insulin resistance and the prevention of type 2 diabetes. J Nutr. 2018;148(1):7-12 https://doi.org/10.1093/jn/nxx008 [published Online First: 2018/01/30].

4. Silva FM, Kramer CK, de Almeida JC, et al. Fiber intake and glycemic control in patients with type 2 diabetes mellitus: a systematic review with metaanalysis of randomized controlled trials. Nutr Rev. 2013;71(12):790-801. https://doi.org/10.1111/nure.12076 [published Online First: 2013/11/05].

5. Francelino Andrade E, Vieira Lobato R, Vasques Araujo T, et al. Effect of beta-glucans in the control of blood glucose levels of diabetic patients: a systematic review. Nutr Hosp. 2014;31(1):170-7. https://doi.org/10.3305/nh. 2015.31.1.7597 [published Online First: 2015/01/07].

6. Gemen R, de Vries JF, Slavin JL. Relationship between molecular structure of cereal dietary fiber and health effects: focus on glucose/insulin response and gut health. Nutr Rev. 2011;69(1):22-33. https://doi.org/10.1111/j.17534887.2010.00357.x [published Online First: 2011/01/05].

7. Papathanasopoulos A, Camilleri M. Dietary fiber supplements: effects in obesity and metabolic syndrome and relationship to gastrointestinal functions. Gastroenterology. 2010;138(1):65-72.e1-2. https://doi.org/10.1053/ j.gastro.2009.11.045 [published Online First: 2009/11/26].

8. Thomson P, Medina DA, Ortuzar V, et al. Anti-inflammatory effect of microbial consortia during the utilization of dietary polysaccharides. Foodserv Res Int (Ottawa, Ont). 2018;109:14-23. https://doi.org/10.1016/j. foodres.2018.04.008 [published Online First: 2018/05/29].

9. Nansel TR, Lipsky LM, Liu A. Greater diet quality is associated with more optimal glycemic control in a longitudinal study of youth with type 1 diabetes. Am J Clin Nutr. 2016;104(1):81-7. https://doi.org/10.3945/ajcn.115. 126136 [published Online First: 2016/05/20].

10. Soedamah-Muthu SS, Chaturvedi N, Fuller JH, et al. Do European people with type 1 diabetes consume a high atherogenic diet? 7-year follow-up of the EURODIAB prospective complications study. Eur J Nutr. 2013;52(7):170110. https://doi.org/10.1007/s00394-012-0473-7 [published Online First: 2012/ 12/12].

11. Balk SN, Schoenaker DA, Mishra GD, et al. Association of diet and lifestyle with glycated haemoglobin in type 1 diabetes participants in the EURODIAB prospective complications study. Eur J Clin Nutr. 2016;70(2):22936. https://doi.org/10.1038/ejcn.2015.110 [published Online First: 2015/07/ 16].

12. Lamichhane AP, Crandell JL, Jaacks LM, et al. Longitudinal associations of nutritional factors with glycated hemoglobin in youth with type 1 diabetes: the SEARCH nutrition ancillary study. Am J Clin Nutr. 2015;101(6):1278-85. https://doi.org/10.3945/ajcn.114.103747 [published Online First: 2015/05/08].

13. Alman AC, Maahs DM, Rewers MJ, et al. Ideal cardiovascular health and the prevalence and progression of coronary artery calcification in adults with and without type 1 diabetes. Diabetes Care. 2014;37(2):521-8. https://doi. org/10.2337/dc13-0997 [published Online First: 2013/10/17].

14. Snell-Bergeon JK, Chartier-Logan C, Maahs DM, et al. Adults with type 1 diabetes eat a high-fat atherogenic diet that is associated with coronary artery calcium. Diabetologia. 2009;52(5):801-9. https://doi.org/10.1007/ s00125-009-1280-4 [published Online First: 2009/02/17].

15. Rimm EB, Giovannucci EL, Stampfer MJ, et al. Reproducibility and validity of an expanded self-administered semiquantitative food frequency questionnaire among male health professionals. Am J Epidemiol. 1992; 135(10):1114-26 discussion 27-36. [published Online First: 1992/05/15].

16. Willett WC, Sampson L, Stampfer MJ, et al. Reproducibility and validity of a semiquantitative food frequency questionnaire. Am J Epidemiol. 1985; 122(1):51-65 [published Online First: 1985/07/01].

17. Willett WC, Sampson L, Browne ML, et al. The use of a self-administered questionnaire to assess diet four years in the past. Am J Epidemiol. 1988; 127(1):188-99 [published Online First: 1988/01/01].

18. Davis PJ, Liu M, Sherman S, et al. HbA1c, lipid profiles and risk of incident type 2 diabetes in United States Veterans. PLoS One. 2018;13(9):e203484. https://doi.org/10.1371/journal.pone.0203484 [published Online First: 2018/ 09/14].

19. Wang $X$, Jung M, Mossavar-Rahmani $Y$, et al. Macronutrient intake, diagnosis status, and glycemic control among US Hispanics/Latinos with diabetes. J Clin Endocrinol Metab. 2016;101(4):1856-64. https://doi.org/10.1210/jc.20153237 [published Online First: 2016/03/08].

20. Lind M, Svensson AM, Kosiborod M, et al. Glycemic control and excess mortality in type 1 diabetes. N Engl J Med. 2014;371(21):1972-82. https:// doi.org/10.1056/NEJMoa1408214 [published Online First: 2014/11/20].

21. Nystrom T, Holzmann MJ, Eliasson B, et al. Glycemic control in type 1 diabetes and long-term risk of cardiovascular events or death after coronary artery bypass grafting. J Am Coll Cardiol. 2015;66(5):535-43. https://doi.org/ 10.1016/j.jacc.2015.05.054 [published Online First: 2015/08/01].

22. McGill CR, Fulgoni VL 3rd, Devareddy L. Ten-year trends in fiber and whole grain intakes and food sources for the United States population: national health and nutrition examination survey 2001-2010. Nutrients. 2015;7(2): 1119-30. https://doi.org/10.3390/nu7021119 [published Online First: 2015/ 02/12].

23. Mehta SN, Volkening LK, Quinn $\mathrm{N}$, et al. Intensively managed young children with type 1 diabetes consume high-fat, low-fiber diets similar to age-matched controls. Nutr Res (New York, NY). 2014;34(5):428-35. https:// doi.org/10.1016/.nutres.2014.04.008 [published Online First: 2014/06/12].

24. Buyken $A E$, Toeller $M$, Heitkamp $G$, et al. Relation of fibre intake to $\mathrm{HbA} 1 \mathrm{C}$ and the prevalence of severe ketoacidosis and severe hypoglycaemia. EURODIAB IDDM complications study group. Diabetologia. 1998:41(8):88290 [published Online First: 1998/09/03].

25. Katz ML, Mehta S, Nansel T, et al. Associations of nutrient intake with glycemic control in youth with type 1 diabetes: differences by insulin regimen. Diabetes Technol Ther. 2014;16(8):512-8. https://doi.org/10.1089/ dia.2013.0389 [published Online First: 2014/04/29].

26. Akturk HK, Garg S. Technological advances shaping diabetes care. Curr Opin Endocrinol Diabetes Obes. 2019. https://doi.org/10.1097/med. 0000000000000467 [published Online First: 2019/02/06].

27. Muller M, Canfora EE, Blaak EE. Gastrointestinal transit time, glucose homeostasis and metabolic health: modulation by dietary fibers. Nutrients. 
2018;10(3). https://doi.org/10.3390/nu10030275 [published Online First: 2018/03/03].

28. Korcz E, Kerenyi Z, Varga L. Dietary fibers, prebiotics, and exopolysaccharides produced by lactic acid bacteria: potential health benefits with special regard to cholesterol-lowering effects. Food Funct. 2018;9(6):3057-68. https://doi.org/10.1039/c8fo00118a [published Online First: 2018/05/24].

29. Wannamethee SG, Whincup PH, Thomas MC, et al. Associations between dietary fiber and inflammation, hepatic function, and risk of type 2 diabetes in older men: potential mechanisms for the benefits of fiber on diabetes risk. Diabetes Care. 2009;32(10):1823-5. https://doi.org/10.2337/dc09-0477 [published Online First: 2009/07/25].

30. Wei B, Liu Y, Lin X, et al. Dietary fiber intake and risk of metabolic syndrome: a meta-analysis of observational studies. Clin Nutr (Edinburgh, Scotland). 2017. https://doi.org/10.1016/j.clnu.2017.10.019 [published Online First: 2017/11/16]

31. Lie L, Brown $L$, Forrester TE, et al. The association of dietary fiber intake with cardiometabolic risk in four countries across the epidemiologic transition. Nutrients. 2018;10(5). https://doi.org/10.3390/nu10050628 [published Online First: 2018/05/19].

32. Grooms KN, Ommerborn MJ, Pham DQ, et al. Dietary fiber intake and cardiometabolic risks among US adults, NHANES 1999-2010. Am J Med. 2013;126(12):1059-67.e1-4. https://doi.org/10.1016/j.amjmed.2013.07.023 [published Online First: 2013/10/19].

33. Ning H, Van Horn L, Shay CM, et al. Associations of dietary fiber intake with long-term predicted cardiovascular disease risk and C-reactive protein levels (from the National Health and nutrition examination survey data [20052010]). Am J Cardiol. 2014;113(2):287-91. https://doi.org/10.1016/j.amjcard. 2013.09.020 [published Online First: 2013/11/02].

34. van Bussel BC, Soedamah-Muthu SS, Henry RM, et al. Unhealthy dietary patterns associated with inflammation and endothelial dysfunction in type 1 diabetes: the EURODIAB study. Nutr Metab Cardiovasc Dis. 2013;23(8):75864. https://doi.org/10.1016/j.numecd.2012.04.005 [published Online First: 2012/07/17].

35. Thompson SV, Hannon BA, An R, et al. Effects of isolated soluble fiber supplementation on body weight, glycemia, and insulinemia in adults with overweight and obesity: a systematic review and meta-analysis of randomized controlled trials. Am J Clin Nutr. 2017;106(6):1514-28. https:// doi.org/10.3945/ajcn.117.163246 [published Online First: 2017/11/03].

36. Stewart ML, Zimmer JP. Postprandial glucose and insulin response to a high-fiber muffin top containing resistant starch type 4 in healthy adults: a double-blind, randomized, controlled trial. Nutrition (Burbank, Los Angeles County, Califf. 2018;53:59-63. https://doi.org/10.1016/.jnut.2018.01.002 [published Online First: 2018/04/16].

37. Beck RW, Riddlesworth T, Ruedy K, et al. Effect of continuous glucose monitoring on glycemic control in adults with type 1 diabetes using insulin injections: the DIAMOND randomized clinical trial. JAMA. 2017;317(4):371-8. https://doi.org/10.1001/jama.2016.19975 [published Online First: 2017/01/25].

Ready to submit your research? Choose BMC and benefit from:

- fast, convenient online submission

- thorough peer review by experienced researchers in your field

- rapid publication on acceptance

- support for research data, including large and complex data types

- gold Open Access which fosters wider collaboration and increased citations

- maximum visibility for your research: over $100 \mathrm{M}$ website views per year

At $\mathrm{BMC}$, research is always in progress.

Learn more biomedcentral.com/submissions 